\title{
Accelerating Existing Non-Blind Image Deblurring Techniques through a Strap-On Limited-Memory Switched Broyden Method
}

\author{
Ichraf LAHOULI $^{\left.\dagger, \dagger+, \dagger \dagger{ }^{\dagger a}\right)}$, Member, Robby HAELTERMAN ${ }^{\dagger}$, Joris DEGROOTE $^{\dagger \dagger \dagger \dagger}$, Michal SHIMONI ${ }^{\dagger}$, \\ Geert DE CUBBER ${ }^{\dagger}$, and Rabah ATTIA ${ }^{\dagger \dagger \dagger}$, Nonmembers
}

\begin{abstract}
SUMMARY Video surveillance from airborne platforms can suffer from many sources of blur, like vibration, low-end optics, uneven lighting conditions, etc. Many different algorithms have been developed in the past that aim to recover the deblurred image but often incur substantial CPU-time, which is not always available on-board. This paper shows how a "strap-on" quasi-Newton method can accelerate the convergence of existing iterative methods with little extra overhead while keeping the performance of the original algorithm, thus paving the way for (near) real-time applications using on-board processing.

key words: image deblurring, quasi-Newton, limited-memory, switched Broyden method
\end{abstract}

\section{Introduction}

Computer vision is an ever expanding scientific domain that has become an integral part of everyday life, be it in surveillance, social, medical or artificial intelligence applications. The images that are taken are often part of a continuous video stream and are seldom an end in itself but serve as a tool for abnormal event detection, navigation, diagnosis, etc. Embedded cameras are often of low quality and can suffer from lens distortion or from vibrations that are transmitted by the platform on which the camera is mounted, like a car or an Unmanned Aerial Vehicle (UAV). The images are therefor corrupted by (motion) blur and noise. Recovering the unblurred image is thus a part of the pre-processing steps that are applied to computer vision applications.

Literally hundreds of publications have appeared over the years (e.g. [4], [14], [19], [36], [55], [56] to name but a few). In particular, when the image blur is spatially invariant then the deblurring process can be seen as a deconvolution problem which is generally ill-conditioned [10], [12], [50]. For this reason the algorithms are generally iterative methods that, unfortunately, require a substantial runtime.

In this paper we present a robust acceleration technique that can be "strapped on" existing iterative algorithms, without the need for delicate tuning of preconditioning

\footnotetext{
Manuscript received September 7, 2017.

Manuscript revised November 13, 2017.

Manuscript publicized February 16, 2018.

$\dagger$ The authors are with Royal Military Academy, Belgium.

${ }^{\dagger \dagger}$ The author is with VRIT Research Group, Military Academy of Tunisia, Tunisia.

${ }^{\dagger \dagger}$ The authors are with SERCOM Lab, Tunisia Polytechnic

$+t_{\dagger}$ The author is with Dept. Flow, Heat \& Combustion Mechanics, Ghent University, Belgium.

a) E-mail: ichraf.lahouli@rma.ac.be DOI: 10.1587/transinf.2017MVP0022
} School, Tunisia. parameters. Besides robustness, the methods offer a typical gain of 5 to $90 \%$ in CPU time compared to a wide range of methods that are known from literature.

This paper is organized as follows. In Sect. 2 we describe the mathematical process behind blurring, while in Sect. 3 we describe existing non-blind iterative algorithms for deblurring images suffering from spatially invariant blur. In Sect. 4 we propose an acceleration technique based on quasi-Newton methods. The convergence speed of the different algorithms is compared in Sect. 5, after which we end with conclusions and ideas for future work.

\section{Blurring Model}

A simplified linear, spatially invariant model of the discrete (i.e. digital) blurring process of an $m \times n$ pixel image is given by

$$
g=K f+\eta
$$

where $f \in \mathbb{R}^{m n}$ is the original image*,$g \in \mathbb{R}^{m n}$ is the blurred image, $\eta \in \mathbb{R}^{m n}$ is additive (most often white Gaussian) noise and $K \in \mathbb{R}^{m n \times m n}$ represents the point spread function $(\mathrm{PSF})^{* *}$ responsible for the actual blurring.

Non-blind image restoration, or deblurring, is the process of finding $f$ based on (approximate) knowledge of $K, n$ and $g$ [33].

The following characteristics make deblurring challenging:

- The problem has a very high dimensionality, which requires matrix-free implementations (cf. Sect. 4.1.4).

- $K$ is, in general, a very ill-conditioned matrix with a cluster of very small singular values.

The effect of the ill-conditioning can be mitigated by the use of regularization to avoid computing solutions that are corrupted by noise. Well-known examples are Tikhonov regularization and Wiener filtering or Total Variation techniques [13], [18], [24], [27], [28], [37], [43], [45], [51]-[54].

Although this approach works well for well-posed problems [3], [23], [46], it does not for ill-posed problems such as image restoration. In this situation, the choice of preconditioner is very sensitive and can result in fast, but

\footnotetext{
${ }^{*}$ Note that we have vectorized the $m \times n$ image by stacking the columns.

** Or "kernel".
} 
erratic, convergence to a poor approximate solution [25], [26], [38]. Furthermore, for these pre-conditioning methods, choosing the best value of the regularization parameter is a nontrivial matter and it may be necessary to solve the problem for many different parameters to determine which is best.

Due to these constraints, iterative image restoration algorithms that do not require preconditioning have become a method of choice, as they have many advantages over simple filtering techniques and can be very efficient for spatially invariant as well as spatially variant blurs [5], [32], [54].

In this paper we focus on the non-blind deconvolution (or deblurring) process and do not take the noise into consideration. We refrain from using pre-conditioning techniques that are based on parameter estimation and in this stage of our research we focus only on spatially invariant blurs.

Our aim is to reduce the cost of an iterative algorithm, which is the product of the CPU time required per iteration and the number of iterations needed to obtain the required quality of the deblurred image.

\section{Deblurring Algorithms}

Preliminary note: for all the algorithms mentioned in this section, we use the implementation found in [4].

Note that these algorithms do not take $\eta$ into account. If (an estimate of) $\eta$ is known it can be catered for by replacing $g$ with $g-\eta$.

\subsection{Landweber Iteration}

The basic Landweber iteration [18], [27], [28], [54], in the context of image restoration, is given by

\section{Landweber iteration}

1. Startup: Take initial value $f_{1}$, set $s=1$.

2. Loop until $\left\|g-K f_{s}\right\|_{2} \leq \epsilon$ :

$$
f_{s+1}=f_{s}+\tau K^{T}\left(g-K f_{s}\right) \text {; set } s=s+1 \text {. }
$$

A typical choice for $\tau$ is $\frac{1}{\sigma_{\max }^{2}}$, where $\sigma_{\max }$ is the largest singular value of $K$, estimated by $\sigma_{\max }=\sqrt{\|K\|_{1}\|K\|_{\infty}}[22]$.

\subsection{Steepest Descent (SD)}

As in the case of the Landweber iteration, the Steepest Descent method takes $K^{T}\left(g-K f_{s}\right)$ as a step direction, but with a variable step-size [4].

Steepest descent
1. Startup: Take initial value $f_{1}$, set $s=1$.
2. Loop until $\left\|g-K f_{s}\right\|_{2} \leq \epsilon$ :
2.1. $f_{s+1}=f_{s}+\frac{\left\|K^{T}\left(g-K f_{s}\right)\right\|_{2}^{2}}{\left\|K K^{T}\left(g-K f_{s}\right)\right\|_{2}^{2}}\left(K^{T}\left(g-K f_{s}\right)\right)$
2.2. Set $s=s+1$

\subsection{LSQR and CGLS}

LSQR and CGLS are related conjugate gradient methods. We refer to the literature for more details [7], [41], [42].

\subsection{Hybrid Method (HM)}

Hybrid methods combine variational approaches with iterative methods, i.e. an iterative conjugate gradient method is applied to $\min \|K f-g\|$ and variational regularization is incorporated in the iterations. Again, we refer to the literature for more details [6], [40].

\subsection{MRNSD and Richardson-Lucy}

As pixels represent perceived intensity values, nonnegativity constraints can be added to the optimisation statement, i.e. we may want to develop algorithms that solve $\min _{f \geq 0}\|K f-g\|$.

Two common algorithms for image restoration with nonnegativity constraints are MRNSD [2], [30], [39] and Richardson-Lucy [33], [44], [54].

\section{Acceleration by Quasi-Newton Methods}

The Landweber iteration in Sect. 3.1 can be seen as a fixedpoint process, where the creation of $f_{s+1}$ based on $f_{s}$ is written as $f_{s+1}=H\left(f_{s}\right)$. Then the problem of finding $f$ can be transformed into a root finding problem $H(f)-f=P(f)=$ 0 . This novel interpretation of deblurring as a root-finding exercise leads us to contemplate the use of a quasi-Newton method as an acceleration method.

\section{Quasi-Newton accelerated Landweber iteration}

1. Startup: Take initial value $f_{1}$, set $s=1$.

2. Loop until $\left\|g-K f_{s}\right\|_{2} \leq \epsilon$ :

2.1. Compute approximate Jacobian $\hat{P}_{s}^{\prime}$ (see below).

2.2. $f_{s+1}=f_{s}-\left(\hat{P}_{s}^{\prime}\right)^{-1} \underbrace{\left(K^{T}\left(g-K f_{s}\right)\right)}_{P\left(f_{s}\right)}$

2.3. Set $s=s+1$

Alternatively a slightly different quasi-Newton step $f_{s+1}=$ $f_{s}-M_{s}^{\prime} P\left(f_{s}\right)$ can be used. Here $M_{s}^{\prime}$ serves as an approximation to the inverse of the Jacobian at step $s$, whereas $\hat{P}_{s}^{\prime}$ is an approximation of the Jacobian itself.

The difference between the various quasi-Newton methods that we consider here lies in the choice of $\hat{P}_{s}^{\prime}$ (or $\left.M_{s}^{\prime}\right)$.

\subsection{Broyden's Methods}

We choose Broyden's methods as the quasi-Newton methods that we will use for the acceleration step, mainly because they have been well-studied and allow for a limitedmemory implementation later on.

To construct the approximate (inverse) Jacobians in 
Broyden's method we first define $\delta f_{s}=f_{s+1}-f_{s}$ and $\delta P_{s}=P\left(f_{s+1}\right)-P\left(f_{s}\right)$.

\subsubsection{Broyden's Good Method}

Broyden's first or good method ${ }^{\dagger}$ (also abbreviated as "BG") [8], [9], [16], [17] is a quasi-Newton method that is part of the family of Least Change Secant Update (LCSU) methods [17], [20], where the approximate Jacobian $\hat{P}_{s+1}^{\prime}$ is chosen as the solution of the following minimization problem:

$$
\min _{\hat{P}^{\prime}}\left\{\left\|\hat{P}^{\prime}-\hat{P}_{s}^{\prime}\right\|_{F r}\right\} \text {, s.t. } \hat{P}^{\prime} \delta f_{s}=\delta P_{s} .
$$

In other words, it gives a new approximate Jacobian that is closest to the previous one in the Frobenius norm and that satisfies the secant equation.

The solution of (2) leads to the following rank-one update:

$$
\hat{P}_{s+1}^{\prime}=\hat{P}_{s}^{\prime}+\frac{\left(\delta P_{s}-\hat{P}_{s}^{\prime} \delta f_{s}\right) \delta f_{s}^{T}}{\left\langle\delta f_{s}, \delta f_{s}\right\rangle}
$$

$\hat{P}_{1}^{\prime}$ is typically set to be equal to $-I$, which means that the first iteration equals a Landweber iteration. Interpreting Broyden's good method differently, we could say that

- $\hat{P}_{s+1}^{\prime}$ is the projection w.r.t. the Frobenius norm of $\hat{P}_{s}^{\prime}$ onto $\left\{A \in \mathbb{R}^{m n \times m n}: A \delta f_{s}=\delta P_{s}\right\}$;

- no change occurs between $\hat{P}_{s+1}^{\prime}$ and $\hat{P}_{s}^{\prime}$ on the orthogonal complement of $\delta f_{s}$, i.e. $\left(\hat{P}_{s+1}^{\prime}-\hat{P}_{s}^{\prime}\right) z=0$ if $\left\langle z, \delta f_{s}\right\rangle=0$.

We have the following properties of this method:

1. For linear problems, the method is known to show superlinear convergence [31] and it needs at most $2 m n$ iteration to reach the solution (Gay's theorem [21]).

2. No guarantee can be given that the approximate Jacobians are non-singular nor that convergence is monotone.

Using using the Sherman-Morrison theorem [49], Broyden's good method can be written as:

$$
\begin{aligned}
& \left(\hat{P}_{s+1}^{\prime}\right)^{-1}= \\
& \quad\left(\hat{P}_{s}^{\prime}\right)^{-1}+\frac{\left(\delta f_{s}-\left(\hat{P}_{s}^{\prime}\right)^{-1} \delta P_{s}\right) \delta f_{s}^{T}\left(\hat{P}_{s}^{\prime}\right)^{-1}}{\delta f_{s}^{T}\left(\hat{P}_{s}^{\prime}\right)^{-1} \delta P_{s}} .
\end{aligned}
$$

\subsubsection{Broyden's Bad Method}

Broyden's second or bad method (also abbreviated as "BB") [8] is a quasi-Newton method that uses an approximation $\hat{M}^{\prime}$ of the inverse Jacobian. It is also part of the family of LCSU methods [17], [20], where the approximate inverse Jacobian $\hat{M}_{s+1}^{\prime}$ is chosen as the solution of the following minimization problem:

\footnotetext{
${ }^{\dagger}$ Most often simply called Broyden's method.
}

$$
\min _{\hat{M}^{\prime}}\left\{\left\|\hat{M}^{\prime}-\hat{M}_{s}^{\prime}\right\|_{F r}\right\} \text {, s.t. } \hat{M}^{\prime} \delta P_{s}=\delta f_{s} .
$$

i.e. it gives a new approximation of the inverse of the Jacobian that is closest to the previous one in the Frobenius norm and that satisfies the secant equation. date

The solution of (5) leads to the following rank- one up-

$$
\hat{M}_{s+1}^{\prime}=\hat{M}_{s}^{\prime}+\frac{\left(\delta f_{s}-\hat{M}_{s}^{\prime} \delta P_{s}\right) \delta P_{s}^{T}}{\left\langle\delta P_{s}, \delta P_{s}\right\rangle} .
$$

Interpreting Broyden's bad method differently, we could say that

- $\hat{M}_{s+1}^{\prime}$ is the projection w.r.t. the Frobenius norm of $\hat{M}_{s}^{\prime}$ onto $\left\{A \in \mathbb{R}^{m n \times m n}: A \delta P_{s}=\delta f_{s}\right\}$;

- no change occurs between $\hat{M}_{s+1}^{\prime}$ and $\hat{M}_{s}^{\prime}$ on the orthogonal complement of $\delta P_{s}$, i.e. $\left(\hat{M}_{s+1}^{\prime}-\hat{M}_{s}^{\prime}\right) z=0$ if $\left\langle z, \delta P_{s}\right\rangle=0$.

Broyden himself [8] admitted that this formulation of his algorithm didn't function properly ${ }^{\dagger \dagger}$. The reasons for the "good" or "bad" behavior are not well understood, and it is quite possible that in some instances the bad method outperforms the good method. Indeed, as we will see in Sect. 5.2, BB performs better than BG in this application. We also have the same properties as for Broyden's Good method.

Even though it is the inverse Jacobian that is approximated in this method, we will write $\left(\hat{P}_{s}^{\prime}\right)^{-1}$ instead of $\hat{M}_{s}^{\prime}$ to standardize the notation of the methods in what follows, thus obtaining

$$
\left(\hat{P}_{s+1}^{\prime}\right)^{-1}=\left(\hat{P}_{s}^{\prime}\right)^{-1}+\frac{\left(\delta f_{s}-\left(\hat{P}_{s}^{\prime}\right)^{-1} \delta P_{s}\right) \delta P_{s}^{T}}{\delta P_{s}^{T} \delta P_{s}} .
$$

Again, $\hat{P}_{1}^{\prime}$ is typically set to be equal to $-I$, which means that the first iteration equals a Landweber iteration.

\subsubsection{Switched Broyden Method}

As Broyden's Good method is not always better than Broyden's Bad method, we follow an idea suggested in [34] that avoids the need to choose between the two methods and create a switched version of BG/BB (called "BS") in the following manner. If

$$
\frac{\left|\delta f_{s}^{T} \delta f_{s-1}\right|}{\left|\delta f_{s}^{T}\left(\hat{P}_{s}^{\prime}\right)^{-1} \delta P_{s}\right|}<\frac{\left|\delta P_{s}^{T} \delta P_{s-1}\right|}{\delta P_{s}^{T} \delta P_{s}}
$$

then the rank-one update of $\mathrm{BG}$ is used, otherwise the update of BB is used. To our knowledge, this variant of Broyden's method has not been given the attention that it deserves, even though we will see that it lies at the basis of the best performing algorithm in Sect. 5.

\subsubsection{Matrix-Free and Limited Memory Implementation of Broyden's Algorithms}

All the methods that we have mentioned so far require

\footnotetext{
"This is the reason the method is called "bad".
} 
matrix-vector multiplications with $K$ and/or $K^{T}$. As the size of the matrices and vectors in the test-cases are typically very large, a matrix-free implementation of the algorithms is necessary. We first develop Broyden's good method as

$$
\left(\hat{P}_{s+1}^{\prime}\right)^{-1}=\left(\hat{P}_{1}^{\prime}\right)^{-1}+\sum_{i=1}^{s} \frac{\delta f_{i}-\left(\hat{P}_{i}^{\prime}\right)^{-1} \delta P_{i}}{\delta f_{i}^{T}\left(\hat{P}_{i}^{\prime}\right)^{-1} \delta P_{i}} \delta f_{i}^{T}\left(\hat{P}_{i}^{\prime}\right)^{-1},
$$

while for Broyden's bad method we get

$$
\left(\hat{P}_{s+1}^{\prime}\right)^{-1}=\left(\hat{P}_{1}^{\prime}\right)^{-1}+\sum_{i=1}^{s} \frac{\left(\delta f_{i}-\left(\hat{P}_{i}^{\prime}\right)^{-1} \delta P_{i}\right)}{\delta P_{i}^{T} \delta P_{i}} \delta P_{i}^{T}
$$

In summary, both methods can be written as

$$
\left(\hat{P}_{s+1}^{\prime}\right)^{-1}=\left(\hat{P}_{1}^{\prime}\right)^{-1}+\sum_{i=1}^{s} w_{i} v_{i}^{T}
$$

where $w_{i}=\delta f_{i}-\left(\hat{P}_{i}^{\prime}\right)^{-1} \delta P_{i}$ and

\begin{tabular}{|c|c|c|}
\hline & BG & BB \\
\hline$v_{i}$ & $\frac{\delta f_{i}^{T}\left(\hat{P}_{i}^{\prime}\right)^{-1}}{\delta f_{i}^{T}\left(\hat{P}_{i}^{\prime}\right)^{-1} \delta P_{i}}$ & $\frac{\delta P_{i}^{T}}{\delta P_{i}^{T} \delta P_{i}}$ \\
\hline
\end{tabular}

Obviously, when a lot of iterations are required, the Broyden methods become both memory heavy and computationally expensive. In this paper we propose a limited memory version of the algorithms, where only the last $\kappa$ $\left(w_{i}, v_{i}\right)$ pairs are kept. We will call the resulting methods $\mathrm{BG}(\kappa), \mathrm{BB}(\kappa)$ and $\mathrm{BS}(\kappa)$ and where the inverse Jacobian is given by

$$
\left(\hat{P}_{s+1}^{\prime}\right)^{-1}=\left(\hat{P}_{1}^{\prime}\right)^{-1}+\sum_{i=\max (1, s-\kappa)}^{s} w_{i} v_{i}^{T} .
$$

Admittedly, the choice of the value $\kappa$ is subject to further research. The only firm conclusion for the moment (as the experiments in the next section will show) is that it cannot be too small (otherwise the method performs badly) nor too high (otherwise the method becomes too computationally heavy).

\section{Performance Comparisons}

\subsection{Test-Cases}

\subsubsection{Spatially Invariant Gaussian Blur}

Gaussian PSFs are typically used to evaluate the performance of deblurring algorithms and can be written as $K=$ $\left[k_{i j}\right]$ with

$$
k_{i j}=\frac{1}{2 \pi \sqrt{\gamma}} e^{-\frac{1}{2}\left([i j] C^{-1}[i j]^{T}\right)}
$$

where

$$
C=\left[\begin{array}{ll}
\alpha_{1}^{2} & \rho^{2} \\
\rho^{2} & \alpha_{2}^{2}
\end{array}\right]
$$
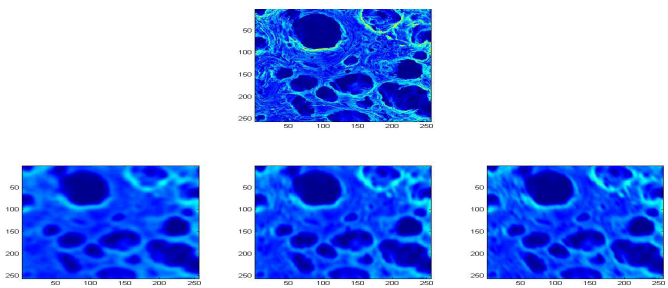

Fig. 1 Gaussian blur. Unblurred image (top), blurred images (bottom), from left to right: G1, G2, G3. $m=n=256$.
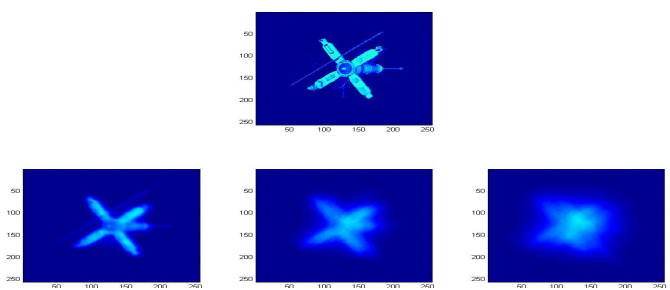

Fig. 2 Atmospheric blur. Unblurred image (top), blurred images (bottom), from left to right: A1, A2, A3. $m=n=256$.

$$
\gamma=\left(\alpha_{1} \alpha_{2}\right)^{2}-\rho^{4}>0
$$

We select three examples of spatially invariant Gaussian blurs from [4] and which are illustrated in Fig. 1:

1. G1: $\left(\alpha_{1}, \alpha_{2}, \rho\right)=(4,4,0)$;

2. G2: $\left(\alpha_{1}, \alpha_{2}, \rho\right)=(4,2,0)$;

3. G3: $\left(\alpha_{1}, \alpha_{2}, \rho\right)=(4,2,2)$.

\subsubsection{Spatially Invariant Atmospheric Turbulence Blur}

When viewing objects through a telescope, a quantification of the observation conditions can be given by $\frac{d}{r_{o}}$, where $d$ is diameter of the telescope and $r_{o}$ is the Fried parameter, which is a statistical measure of atmospheric turbulence [29]. A low value of $\frac{d}{r_{o}}$ corresponds to good conditions.

We use three models, illustrated in Fig. 2, corresponding to $\frac{d}{r_{o}}=10(\mathrm{~A} 1), 30(\mathrm{~A} 2)$ and 50 (A3) respectively.

\subsection{Comparison between Broyden Variants}

We first compare the Landweber iteration with BG, BB and $\mathrm{BS}$. As a convergence criterion $\epsilon=m n \cdot 10^{-3}$ is used. As the experiments will show, this is a rather stringent convergence criterion, corresponding to a high quality of the restored image (e.g. Fig. 3 for G1).

For all six test-cases the same behavior was noted:

- All methods had monotonous convergence, except for Broyden's good method.

- Convergence for Landweber's method was significantly slower than for BB and BS. The performance of the latter two methods was very similar over the range of test-cases.

The convergence history for test-case G1 is given in 

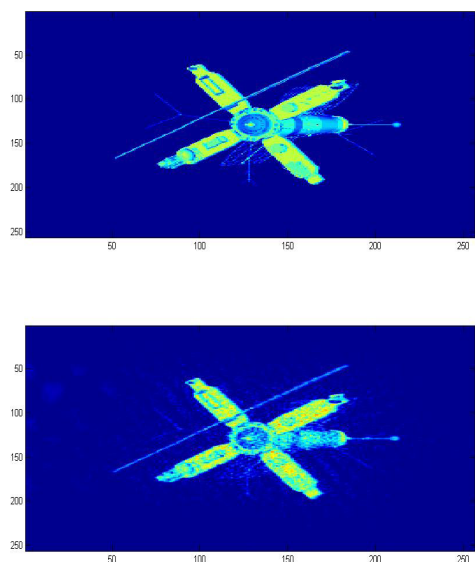

Fig. 3 Atmospheric blur A2. Unblurred image (top), restored image with BS (bottom).
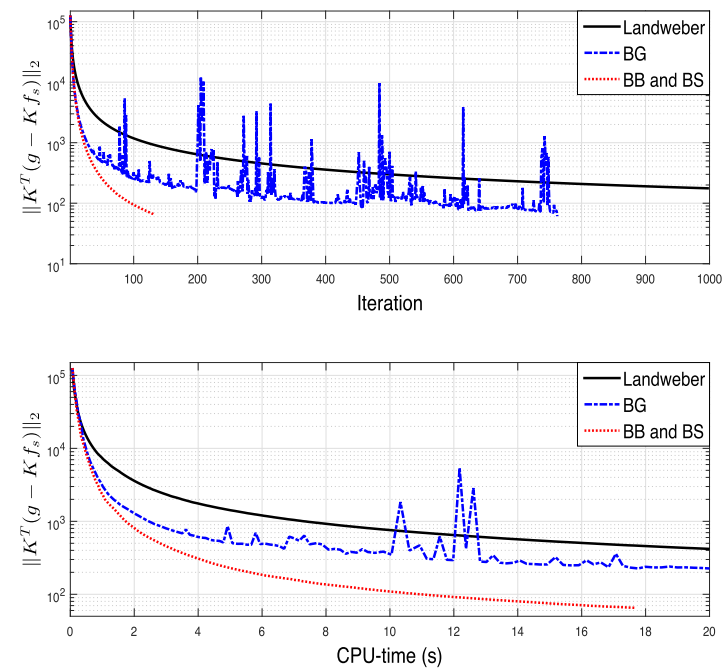

Fig. 4 Convergence history against iteration count and CPU-time for test-case G1 using Landweber, BG, BB and BS.

Fig. 4 (both as a function of iteration count and as a function of CPU time) and in Fig. 5 for test-case A2. Results for BB and BS are indistinguishable on the figures. (Results for test-cases G2 and G3 are very similar to G1, while those for $\mathrm{A} 1$ and $\mathrm{A} 3$ are very similar to A2.) The gain in CPU time (measured on a 3.3GHz Intel Core i3-2120 with 4GB RAM) for BS, compared to Landweber iteration, was in the order of $90 \%$ for all test-cases. Typically the order of convergence (approximated by $\frac{\log \left|\frac{f_{s+1}-f_{s}}{f_{s}-f_{s-1}}\right|}{\log \frac{f_{s-f}-f_{s-1}}{f_{s-1}-f_{s-2}} \mid}$, for a sufficiently big value of $s$ ) of the Landweber method was 0.8 to 0.95 , while that of BS was 1.2 to 1.3 .

Looking at these results, we chose BS as the best method of these four.

When we compare BS with BS( $\kappa)$, we note that for values of $\kappa>10$ (Fig. 6), divergence is possible, although it must be added that for $\kappa=11$ or 12 , this is just barely, as the algorithm comes within a whisker of the (admittedly stringent) stopping criterion before diverging. The source
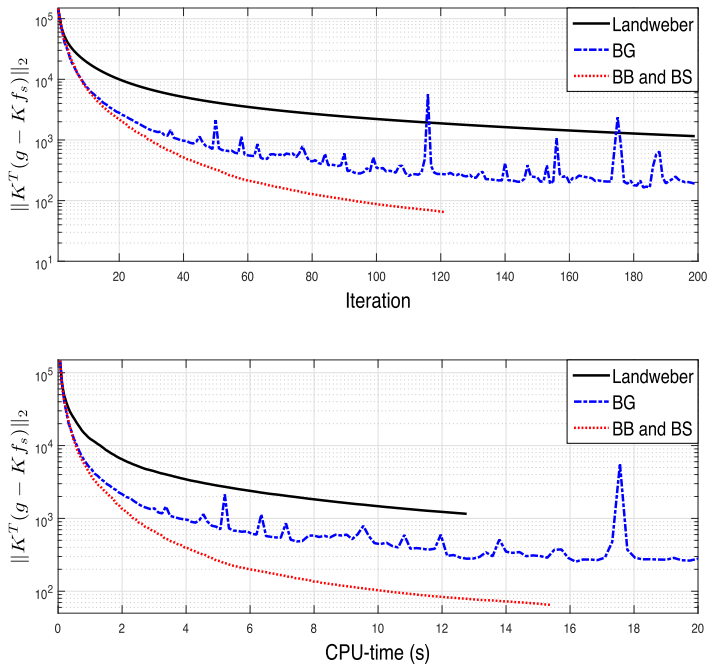

Fig.5 Convergence history against iteration count and CPU-time for test-case A2 using Landweber, BG, BB and BS.
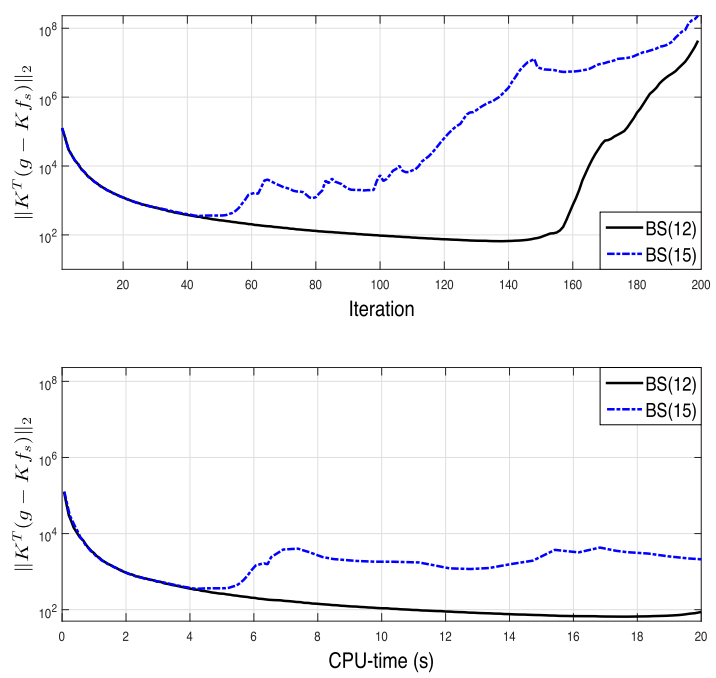

Fig. 6 Convergence history against iteration count and CPU-time for test-case G1 using BS(12) and BS(15).

of this phenomenon is the numerical instability of the chosen limited-memory algorithm. While this might warrant further investigation, it needs to be emphasized that there is no point in trying to keep the algorithm stable for very high values of $\kappa$ as this would go against the idea of a limitedmemory method.

When we compare $\mathrm{BS}$ with $\mathrm{BS}(\kappa)$, we further note that

- using $\kappa \in[2,10]$ does not significantly change the convergence behavior when plotted against iteration number (Fig. 7), with $\kappa=8$ giving slightly better results. In particular, Fig. 7 shows that BS(10) is slightly better than $\mathrm{BS}(8)$ when comparing the iteration count, but slightly worse when comparing the CPU-time. A value of $\kappa=8$ is therefore the one around which the gain in CPU-time per iteration starts to outweigh the gain in total number of iterations. 

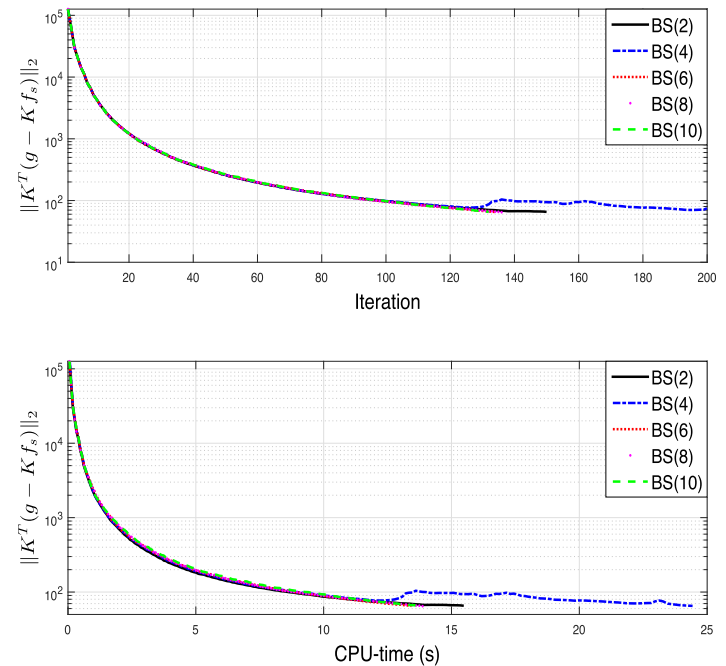

Fig. 7 Convergence history against iteration count and CPU-time for test-case G1 using BS(2), BS(4), BS(6), BS(8) and BS(10).
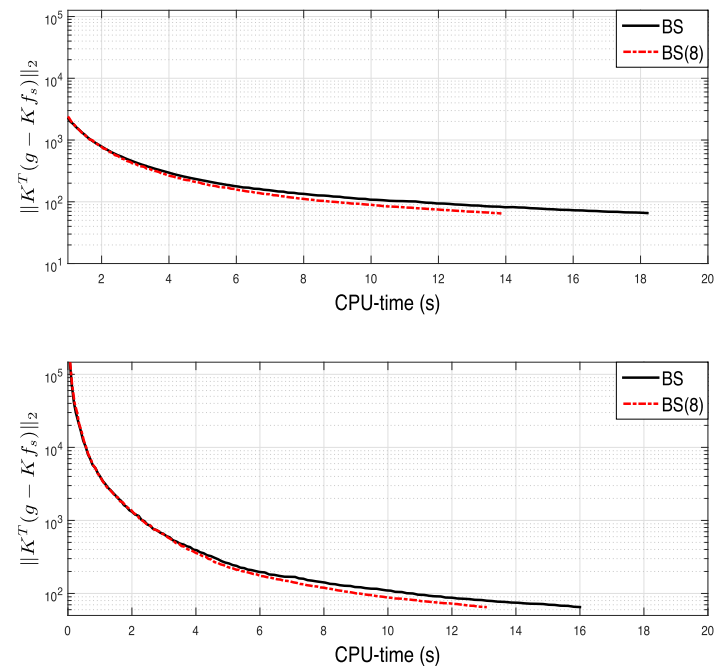

Fig. 8 Convergence history for test-cases G1 (top) and A2 (bottom) using BS and BS(8).

- When plotted against CPU-time BS(8) yielded a gain of roughly $25 \%$ compared to BS on test-case G2 (Fig. 7) and 20\% compared to BS on test-case A2 (Fig. 8). For other blurs the results were similar.

\subsection{Comparison with Other Methods}

Having selected the best-performing Broyden variant (i.e. $\mathrm{BS}(8)$ ), we turn our attention to the more sophisticated iterative methods for non-blind deblurring described in [4] and using the implementations therein. These are "Steepest Descent" (SD), "LSQR", "CGLS", the "Hybrid Method" (HM), MRNSD and Richardson-Lucy. The convergence history for test-cases G1, G2 and A1 are given in Figs. 9 and 10; those for G3, A2 and A3 (not shown) exhibit similar behavior. The best performing of these methods is SD. For the test-cases with Gaussian blur it still required around
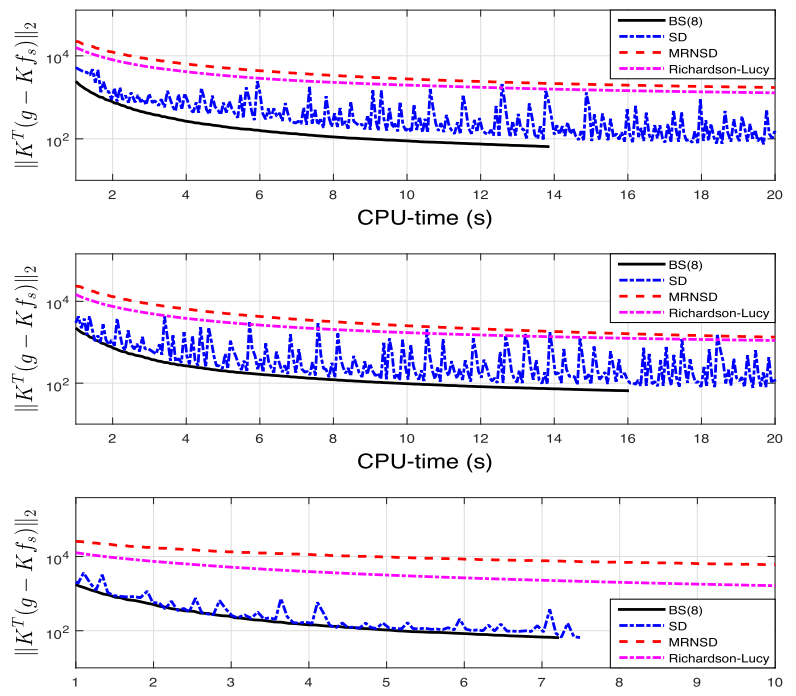

Fig. 9 Convergence history for test-cases G1 (top), G2 (middle) and A1 (bottom) using BS(8), SD, MRNSD and Richardson-Lucy.
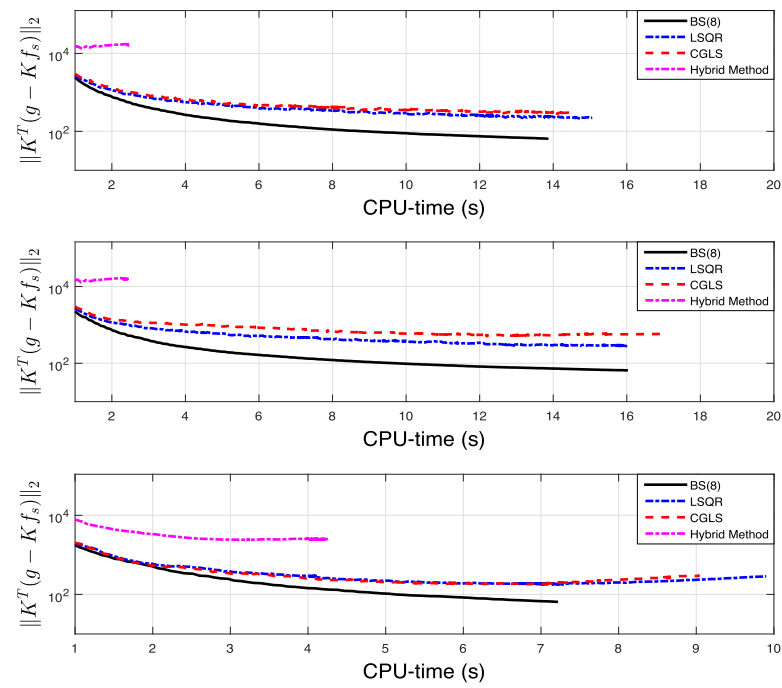

Fig. 10 Convergence history for test-cases G1 (top), G2 (middle) and A1 (bottom) using BS(8), LSQR, CGLS and the Hybrid Method.

$50 \%$ to $70 \%$ more CPU time than BS(8), while for the atmospheric blurring it only required around 5 to $10 \%$ more. Furthermore, convergence of SD is erratic, while BS(8) has monotone convergence. All the other methods initially had a monotone, but excessively slow convergence, which sometimes resulted in stagnation (typical for the Hybrid Method) or in slow divergence before the tolerance was reached. In none of the cases, the performance came close to that of $\mathrm{BS}(8)$.

\section{Conclusions}

We have shown how a switched variant of Broyden's quasiNewton method, and in particular a matrix-free, limited memory version of this algorithm, offers faster convergence than the most common iterative methods used for non-blind 
image deblurring. Compared to the most competitive of the other methods, the gain is still in the order of 5 to $40 \%$ in CPU time for the test-cases that we used. Furthermore the new methods offers monotone convergence and is easy to implement. We would like to point out that at this point the new quasi-Newton method has not yet been computationally optimized and that further gains are possible when tweaked accordingly. This will form the basis of future work.

\section{References}

[1] J.M. Bardsley, "An efficient computational method for total variation-penalized Poisson likelihood estimation," Inverse Problems and Imaging, vol.2, no.2, pp.167-185, 2008.

[2] J.M. Bardsley and J.G. Nagy, "Covariance-preconditioned iterative methods for nonnegatively constrained astronomical imaging," SIAM J. Matrix Anal. Appl., vol.27, no.4, pp.1184-1197, 2006.

[3] M. Benzi, "Preconditioning techniques for large linear systems: A survey," Journal of Computational Physics, vol.182, no.2, pp.418-477, 2002.

[4] S. Berisha and J.G. Nagy, Iterative Methods for Image Restoration. http://www.mathcs.emory.edu/nnagy/RestoreTools/IR.pdf

[5] M. Bertero and P. Boccacci, Introduction to Inverse Problems in Imaging, Institute of Physics Publishing, London, 1998.

[6] Å. Björck, "A bidiagonalization algorithm for solving large and sparse ill-posed systems of linear equations," BIT, vol.28, no.3, pp.659-670, 1988.

[7] ̊.. Björck, Numerical Methods for Least Squares Problems, SIAM, Philadelphia, PA, 1996.

[8] C.G. Broyden, "A class of methods for solving nonlinear simultaneous equations," Math. Comp., vol.19, pp.577-593, 1965.

[9] C.G. Broyden, "Quasi-Newton methods and their application to function minimisation," Math. Comp., vol.21, pp.368-381, 1967.

[10] J.-F. Cai, H. Ji, C. Liu, and Z. Shen, "Framelet-based blind motion deblurring from a single image," IEEE Trans. Image Process., vol.21, no.2, pp.562-Ü572, 2012.

[11] D. Calvetti and E. Somersalo, Introduction to Bayesian Scientific Computing, Springer-Verlag, New York, 2007.

[12] P. Campisi and K. Egiazarian, Blind Image Deconvolution: Theory and Applications, CRC Press, 2007.

[13] T.F. Chan and J. Shen, Image Processing and Analysis: Variational, PDE, Wavelet, and Stochastic Methods, SIAM, Philadelphia, PA, 2005.

[14] X. Chen, X. He, J. Yang, and Q. Wu, "An effective document image deblurring algorithm," 2011 IEEE Conference on Computer Vision and Pattern Recognition (CVPR), pp.369-376, IEEE, 2011.

[15] P.L. Combettes, "The foundations of set theoretic estimation," Proc. IEEE, vol.91, pp.182-208, 1993.

[16] J.E. Dennis and J.J. Moré, "Quasi-Newton methods, motivation and theory," SIAM Rev., vol.19, no.1, pp.46-89, 1977.

[17] J.E. Dennis and R.B. Schnabel, "Least Change Secant Updates for quasi-Newton methods," SIAM Rev., vol.21, no.4, pp.443-459, 1979.

[18] H.W. Engl, M. Hanke, and A. Neubauer, Regularization of Inverse Problems, Kluwer Academic Publishers, Dordrecht, 2000.

[19] G.A. Farulla, M. Indaco, D. Rolfo, L.O. Russo, and P. Trotta, "Evaluation of image deblurring algorithms for real-time applications," 9th IEEE International Conference On Design \& Technology of Integrated Systems In Nanoscale Era (DTIS), Santorini, 6-8 May 2014, pp.1-6, 2014.

[20] A. Friedlander, M.A. Gomes-Ruggiero, D.N. Kozakevich, J.M. Martínez, and S.A. dos Santos, "Solving nonlinear systems of equations by means of quasi-Newton methods with a nonmonotone strategy," Optim. Methods Softw., vol.8, no.1, pp.25-51, 1997.

[21] D.M. Gay, "Some convergence properties of Broyden's method,"
SIAM J. Numer. Anal., vol.16, no.4, pp.623-630, 1979.

[22] G.H. Golub and C. Van Loan, Matrix Computations, 3rd edition, Johns Hopkins Univ. Press, 1996.

[23] A. Greenbaum, Iterative Methods for Solving Linear Systems, SIAM, Philadelphia, 1997.

[24] C.W. Groetsch, The Theory of Tikhonov Regularization for Fredholm Integral Equations of the First Kind, Pitman, Boston, 1984.

[25] M. Hanke, J.G. Nagy, and R.J. Plemmons, "Preconditioned iterative regularization for ill-posed problems," ed. L. Reichel, A. Ruttan, and R.S. Varga, Numerical Linear Algebra, pp.141-163, de Gruyter, Berlin, 1993.

[26] M. Hanke and J.G. Nagy, "Restoration of atmospherically blurred images by symmetric indefinite conjugate gradient techniques," Inverse Problems, vol.12, no.2, pp.157-173, 1996.

[27] P.C. Hansen, Rank-deficient and Discrete Ill-posed Problems, SIAM, Philadelphia, PA, 1997.

[28] P.C. Hansen, Discrete Inverse Problems: Insight and Algorithms, SIAM, Philadelphia, PA, 2010.

[29] S.M. Jefferies and M. Hart, "Deconvolution from wave front sensing using the frozen flow hypothesis," Optics Express, vol.19, no.3, pp.1975-1984, 2011.

[30] L. Kaufman, "Maximum likelihood, least squares, and penalized least squares for PET," IEEE Trans. Med. Imag., vol.12, no.2, pp.200-214, 1993.

[31] C.T. Kelley, Iterative Methods for Linear and Nonlinear Equations, SIAM, Philadelphia, 1995.

[32] R.L. Lagendijk and J. Biemond, Iterative Identification and Restoration of Images, Kluwer Academic Publishers, Boston/Dordrecht/ London, 1991.

[33] L. Lucy, "An iterative technique for the rectification of observed distributions," The astronomical journal, vol.79, p.745, 1974.

[34] J.M. Martínez and L.S. Ochi, "Sobre Dois Metodos de Broyden," Mat. Apl. Comput., vol.1, no.2, pp.135-143, 1982.

[35] J.M. Martínez, "Practical quasi-Newton methods for solving nonlinear systems," J. Comput. Appl. Math., vol.124, no.1-2, pp.97-121, 2000.

[36] Q. Shan, J. Jia, and A. Agarwala, "High-quality motion deblurring from a single image," ACM Transactions on Graphics (TOG), vol.27, p.73, ACM, 2008.

[37] K. Miller, "Least squares methods for ill-posed problems with a prescribed bound," SIAM J. Math. Anal., vol.1, no.1, pp.52-74, 1970.

[38] J.G. Nagy, R.J. Plemmons, and T.C. Torgersen, "Iterative image restoration using approximate inverse preconditioning," IEEE Trans. Image Process., vol.5, no.7, pp.1151-1162, 1996.

[39] J.G. Nagy and Z. Strakoš, "Enforcing nonnegativity in image reconstruction algorithms," ed. D.C. Wilson, et al., Mathematical Modeling, Estimation, and Imaging, vol.3461, pp.182-190, SPIE, 2000.

[40] D.P. O'Leary and J.A. Simmons, "A bidiagonalization-regularization procedure for large scale discretizations of ill-posed problems," SIAM J. Sci. Stat. Comp., vol.2, no.4, pp.474-489, 1981.

[41] C.C. Paige and M.A. Saunders, "LSQR: An algorithm for sparse linear equations and sparse least squares," ACM Trans. Math. Soft., vol.8, no.1, pp.43-71, 1982.

[42] C.C. Paige and M.A. Saunders, "Algorithm 583: LSQR: Sparse linear equations and least squares problems," ACM Trans. Math. Soft., vol.8, no.2, pp.195-209, 1982.

[43] D.L. Phillips, "A technique for the numerical solution of certain integral equations of the first kind," Journal of the Association for Computing Machinery, vol.9, no.1, pp.84-97, 1962.

[44] W.H. Richardson, "Bayesian-based iterative method of image restoration," J. Optical Soc. Amer., vol.62, no.1, pp.55-59, 1972.

[45] L.I. Rudin, S. Osher, and E. Fatemi, "Nonlinear total variation based noise removal algorithms," Physica D, vol.60, no.1-4, pp.259-268, 1992.

[46] Y. Saad, Iterative Methods for Sparse Linear Systems, 2nd Ed., SIAM, Philadelphia, 2003. 
[47] M.I. Sezan and H.J. Trussell, "Prototype image constraints for set-theoretic image restoration," IEEE Trans. Signal Process., vol.39, no.10, pp.2275-2285, 1991.

[48] G. Sharma and H.J. Trussell, "Set theoretic signal restoration using an error in variables criterion," IEEE Trans. Image Process., vol.6, no.12, pp.1692-1697, 1997.

[49] A.H. Sherman and W.J. Morrison, "Adjustment of an inverse matrix corresponding to changes in the elements of a given column or a given row of the original matrix," Ann. Math. Statist., vol.21, pp.124-127, 1950.

[50] M.M. Sondhi, "Image restoration: The removal of spatially invariant degradations," Proc. IEEE, vol.60, no.7, pp.842-853, 1972.

[51] A.N. Tikhonov, "Regularization of incorrectly posed problems," Soviet Math. Doklady, vol.4, pp.1624-1627, 1963.

[52] A.N. Tikhonov, "Solution of incorrectly formulated problems and the regularization method," Soviet Math. Doklady, vol.4, pp.501504, 1963.

[53] A.N. Tikhonov and V.Y. Arsenin, Solutions of Ill-Posed Problems, Winston, Washington, D.C., 1977.

[54] C.R. Vogel, Computational Methods for Inverse Problems, SIAM, Philadelphia, PA, 2002.

[55] W. Wang and M.K. Ng, "On algorithms for automatic deblurring from a single image," Journal of Computational Mathematics, vol.30, no.1, pp.80-100, 2012.

[56] O. Whyte, J. Sivic, A. Zisserman, and J. Ponce, "Non-uniform deblurring for shaken images," International Journal of Computer Vision, vol.98, no.2, pp.168-186, 2012.

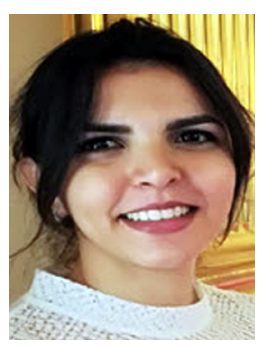

Ichraf Lahouli obtained her M.S degree in Telecommunication engineering from the Military Academy of Tunisia in 2010. She did her master thesis in the Royal Military Academy of Belgium (RMA) about scene classification in an indoor environment. Currently, she is doing a joint $\mathrm{PhD}$ between the RMA and Tunisia Polytechnic School (TPS). She works on abnormal events' detection using UAVs (Unmanned Aerial Vehicles).

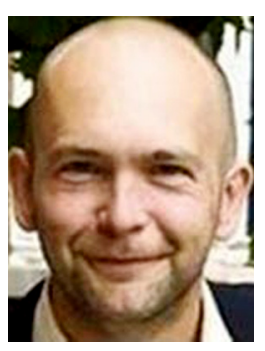

Robby Haelterman obtained his M.S. degree in Engineering from the Royal Military Academy of Belgium in 1998 and his PhD from the Royal Military Academy and the University of Ghent in 2009. He currently works at the Mathematics Department of the Royal Military Academy of Belgium as an Associate Professor.

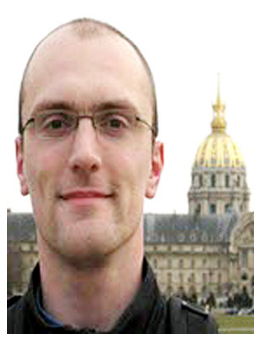

Joris Degroote received the M.S. and Ph.D. degrees in Mechanical Engineering from Ghent University in 2006 and 2010, respectively. During 2007-2008, he stayed at Massachusetts Institute of Technology and in 2011 at Technische Universität München. Currently, he is an associate professor at Ghent University.

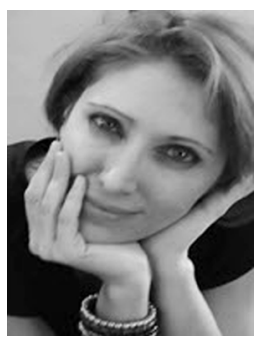

Michal Shimoni received the B.S. and M.S. in geophysics from Tel-Aviv University, Israel in 1993 and 1997 respectively. She obtained her master in civil engineering at the Gembloux University, Belgium in 2000. Michal is a senior researcher and project officer since 1999 at the Signal and Image Centre of the Belgian Royal Military Academy. She has got more than 15 years of experience in the field of image processing and image analysis for geoscience and applications. Her research interests include imaging spectroscopy, PolSAR, PolInSAR, and multi sensor fusion. Since 2009, Michal is an associated editor for IEEE GRSL and external expert for several European Space programmes.

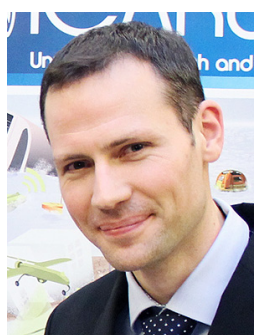

Geert De Cubber obtained his Master in Engineering at the Vrije Universiteit Brussel (VUB), and a PhD. from the Belgian Royal Military Academy (RMA) and VUB for his research in the field of 3-dimensional reconstruction of natural scenes perceived by mobile robots. Currently, he is a researcher working in the department of Mechanics of the Royal Military Academy, where he is leading the research activities of the research group on robotics for high-risk applications. He is also the coordinator of the EU research projects ICARUS (on the development of unmanned search and rescue tools) and SafeShore (on the development of a detection system for threat agents).

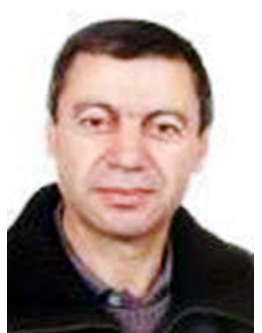

Rabah Attia received his $\mathrm{PhD}$ in telecommunications from the University of Valenciennes, France, in 1986. He is a professor of optical communication at Tunisia Polytechnic School (TPS), Tunisia. Since 2013, he has been the Director for SERCOM Laboratory at TPS, Tunisia. His research interests include new generation of optical fiber, photonic crystal component, electro-optic modulators, and cooperative/relay networks. 